\title{
Hidden Symmetries of Euclideanised Kerr-NUT-(A)dS Metrics in Certain Scaling Limits ${ }^{\star}$
}

\author{
Mihai VISINESCU ${ }^{\dagger}$ and Gabriel Eduard VÎLCU $\ddagger \S$ \\ $\dagger$ National Institute for Physics and Nuclear Engineering, Department of Theoretical Physics, \\ P.O. Box M.G.-6, Magurele, Bucharest, Romania \\ E-mail:mvisin@theory.nipne.ro \\ $\ddagger$ Petroleum-Gas University of Ploieşti, Department of Mathematical Economics, \\ Bulevardul Bucureşti, Nr. 39, Ploieşti 100680, Romania \\ E-mail: gvilcu@upg-ploiesti.ro \\ $\S$ University of Bucharest, Faculty of Mathematics and Computer Science, \\ Research Center in Geometry, Topology and Algebra, \\ Str. Academiei, Nr. 14, Sector 1, Bucharest 70109, Romania \\ E-mail: gvilcu@gta.math.unibuc.ro
}

Received May 29, 2012, in final form July 23, 2012; Published online August 27, 2012

http://dx.doi.org/10.3842/SIGMA.2012.058

\begin{abstract}
The hidden symmetries of higher dimensional Kerr-NUT-(A)dS metrics are investigated. In certain scaling limits these metrics are related to the Einstein-Sasaki ones. The complete set of Killing-Yano tensors of the Einstein-Sasaki spaces are presented. For this purpose the Killing forms of the Calabi-Yau cone over the Einstein-Sasaki manifold are constructed. Two new Killing forms on Einstein-Sasaki manifolds are identified associated with the complex volume form of the cone manifolds. Finally the Killing forms on mixed 3-Sasaki manifolds are briefly described.
\end{abstract}

Key words: Killing forms; Einstein-Sasaki space; Calabi-Yau spaces

2010 Mathematics Subject Classification: 53C15; 53C25; 81T20

\section{Introduction}

The usual spacetimes symmetries are represented by isometries connected with the Killing vector fields. Slightly more generally, the conformal Killing vector field preserve a given conformal class of metrics. For each of the (conformal) Killing vector fields there exists a conserved quantity for the (null) geodesic motions.

Besides them a spacetime may also possess hidden symmetries generated by higher order symmetric or antisymmetric tensor fields. The symmetric Stäckel-Killing tensors give rise to conserved quantities of higher order in particle momenta. A natural generalization of (conformal) Killing vector fields is given by the antisymmetric (conformal) Killing-Yano tensors. Killing-Yano tensors are also called Yano tensors or Killing forms, and conformal Killing-Yano tensors are sometimes referred as conformal Yano tensors, conformal Killing forms or twistor forms.

In physics, Yano tensors play a fundamental role being related to the separability of field equations with spin, pseudoclassical spinning models, the existence of quantum symmetry operators, supersymmetries, etc.

\footnotetext{
*This paper is a contribution to the Special Issue "Geometrical Methods in Mathematical Physics". The full collection is available at http://www.emis.de/journals/SIGMA/GMMP2012.html
} 
In this paper we want to take a closer look at the Killing forms of Kerr-NUT-(A)dS metrics which are related to Einstein-Sasaki metrics. An Einstein-Sasaki manifold is a Riemannian manifold that is both Sasakian and Einstein. In the last time Sasakian manifolds, as an odddimensional analog of Kähler manifolds, have become of high interest. Sasakian manifolds are contact manifolds satisfying a normality (or integrability) condition. On the other hand the contact geometry is motivated by classical mechanics, a contact space corresponding to the odd-dimensional extended phase space that includes the time variable. Recently EinsteinSasaki geometries have been the object of much attention in connection with the supersymmetric backgrounds relevant to the AdS/CFT correspondence. On the other hand a lot of interest focuses on higher dimensional black hole spacetimes [20]. The search of hidden symmetries generated by the Killing forms in rotating black hole geometries has an important role for describing the properties of black holes in various dimensions.

The Kerr-NUT-AdS metrics in all dimensions have been constructed in [16]. The general Kerr-NUT-AdS metrics have $(2 n-1)$ non-trivial parameters where the spacetime dimension is $(2 n+1)$ in the odd-dimensional case and $(2 n)$ in the even dimensional case. It was also considered the BPS, or supersymmetric, limits of these metrics. After Euclideanisation, these limits yield in odd dimensions new families of Einstein-Sasaki metrics, whereas the even-dimensional metrics result in the Ricci-flat Kähler manifolds. An alternative procedure was proposed in [35] generalizing the scaling limit of Martelli and Sparks [38]. More precisely, in a certain limit one gets an Einstein-Kähler metric from an even-dimensional Kerr-NUT-(A)dS spacetime and the Einstein-Sasaki space is constructed as a $U(1)$ bundle over this metric. On the other hand, performing the scaling limit of the odd-dimensional Kerr-NUT-(A)dS spacetimes one gets directly the same Einstein-Sasaki space obtained as a $U(1)$ bundle over the Einstein-Kähler metric [35].

The Kerr-NUT-(A)dS metrics possess explicit and hidden symmetries encoded in a series of rank two Stäckel-Killing tensors and Killing vectors [16]. These symmetries allow one constructs a set of quantities conserved along geodesics. Moreover they are functionally independent and in involution and guarantee complete integrability of the geodesic motions [34, 43, 47].

The structure of the hidden symmetries for a Sasaki space is derived from the characteristic Sasakian 1-form. Killing-Yano tensors alternate closed conformal Killing-Yano tensors as the rank increases [35]. The corresponding hidden symmetries are purely geometrical, irrespective of the fact whether the Einstein equations are satisfied or not.

One of the purposes of this paper is to point out the special case of the higher dimensional Kerr-NUT-(A)dS metrics which are related to the Einstein-Sasaki ones. In this case there are two additional Killing-Yano tensors taking into account that the metric cone is CalabiYau [46]. These two exceptional Killing forms can be also described using the Killing spinors of an Einstein-Sasaki manifold [5].

In the main body of the present paper we consider the Killing forms on Einstein-Sasaki spaces. Let us remark that versions of $M$-theory could be formulated in spacetimes with various number of time dimensions giving rise to exotic spacetime signatures [26, 27]. The paraquaternionic structures arise in a natural way in modern studies in string theories, integrable systems $[17,19,40]$. The counterpart in odd-dimensions of a paraquaternionic structure is called mixed 3-structure which appears in a natural way on lightlike hypersurfaces in paraquaternionic manifolds. For completeness we extend the study of Killing forms on other more particular Sasaki structures.

In Section 2 we review some basic facts about the Einstein-Sasaki spaces and their cone manifolds. In the next section we discuss the Killing forms on Einstein-Sasaki spaces which proceed from Euclideanised Kerr-NUT-(A)dS metrics in certain scaling limits. We identity two new Killing forms associated with the complex volume form of the cone manifold. The paper ends with conclusions in Section 4. In an appendix we briefly discuss the Killing forms on mixed 3-Sasaki manifolds. 


\section{Mathematical preliminaries}

For convenience, the mathematical concepts and results needed to study the hidden symmetries on Einstein-Sasaki spaces are summarized in this Section.

\subsection{Killing vector fields and their generalizations}

A vector field $X$ on a (pseudo-)Riemannian manifold $(M, g)$ is said to be a Killing vector field if the Lie derivative of the metric $g$ with respect to $X$ vanishes or, equivalently, if the Levi-Civita connection $\nabla$ of $g$ satisfies

$$
g\left(\nabla_{Y} X, Z\right)+g\left(Y, \nabla_{Z} X\right)=0,
$$

for all vector fields $Y, Z$ on $M$. A natural generalization of Killing vector fields is given by the conformal Killing vector fields, i.e. vector fields with a flow preserving a given conformal class of metrics [51]. On the other hand, a conformal Killing-Yano tensor of rank $p$ on a (pseudo-) Riemannian manifold $(M, g)$ is a $p$-form $\omega$ which satisfies:

$$
\left.\nabla_{X} \omega=\frac{1}{p+1} X\right\lrcorner d \omega-\frac{1}{n-p+1} X^{*} \wedge d^{*} \omega
$$

for any vector field $X$ on $M$, where $\nabla$ is the Levi-Civita connection of $g, n$ is the dimension of $M, X^{*}$ is the 1 -form dual to the vector field $X$ with respect to the metric $\left.g,\right\lrcorner$ is the operator dual to the wedge product and $d^{*}$ is the adjoint of the exterior derivative $d$. If $\omega$ is co-closed in (1), then we obtain the definition of a Killing-Yano tensor (introduced by Yano [51]). It is easy to see that for $p=1$, they are dual to Killing vector fields. Moreover, a Killing form $\omega$ is said to be a special Killing form if it satisfies for some constant $c$ the additional equation

$$
\nabla_{X}(d \omega)=c X^{*} \wedge \omega
$$

for any vector field $X$ on $M$.

Besides the antisymmetric generalization of the Killing vectors one might also consider higher order symmetric tensors. A symmetric tensor $K_{\left(i_{1} \ldots i_{k}\right)}$ obeying the equation

$$
K_{\left(i_{1} \ldots i_{k} ; j\right)}=0
$$

is called a Stäckel-Killing tensor. For any geodesic with a tangent vector $u^{i}$ the following object

$$
P_{K}=K_{i_{1} \ldots i_{k}} u^{i_{1}} \cdots u^{i_{k}}
$$

is conserved.

These two generalizations of the Killing vectors could be related. Given two Killing-Yano tensors $\omega^{i_{1}, \ldots, i_{r}}$ and $\sigma^{i_{1}, \ldots, i_{r}}$ it is possible to associate with them a Stäckel-Killing tensor of rank 2:

$$
K_{i j}^{(\omega, \sigma)}=\omega_{i i_{2} \ldots i_{r}} \sigma_{j}^{i_{2} \ldots i_{r}}+\sigma_{i i_{2} \ldots i_{r}} \omega_{j}^{i_{2} \ldots i_{r}} .
$$

Therefore a method to generate higher order integrals of motion is to identify the complete set of Killing-Yano tensors. The existence of enough integrals of motion leads to complete integrability or even superintegrability of the mechanical system when the number of functionally independent constants of motion is larger than its number of degrees of freedom. Let us mention that when a Stäckel-Killing tensor is of the form (2), there are no quantum anomalies thanks to an integrability condition satisfied by the Killing-Yano tensors [14]. 


\subsection{Almost Hermitian manifolds and the complex volume form}

An almost (pseudo-)Hermitian structure on a smooth manifold $M$ is a pair $(g, J)$, where $g$ is a (pseudo-)Riemannian metric on $M$ and $J$ is an almost complex structure on $M$, which is compatible with $g$, i.e.

$$
g(J X, J Y)=g(X, Y),
$$

for all vector fields $X, Y$ on $M$. In this case, the triple $(M, J, g)$ is called an almost (pseudo-)Hermitian manifold. Moreover, if $J$ is parallel with respect to the Levi-Civita connection $\nabla$ of $g$, then $(M, J, g)$ is said to be a Kähler manifold (with indefinite metric). We remark that on a Kähler manifold, the associated Kähler form, i.e. the alternating 2-form $\Omega$ defined by

$$
\Omega(X, Y)=g(J X, Y),
$$

is closed. In local holomorphic coordinates $\left(z^{1}, \ldots, z^{m}\right)$, the associated Kähler form $\Omega$ can be written as

$$
\Omega=i g_{j \bar{k}} d z^{j} \wedge d \bar{z}^{k}=\sum X_{j}^{*} \wedge Y_{j}^{*}=\frac{i}{2} \sum Z_{j}^{*} \wedge \bar{Z}_{j}^{*},
$$

where $\left(X_{1}, Y_{1}, \ldots, X_{m}, Y_{m}\right)$ is an adapted local orthonormal field (i.e. such that $Y_{j}=J X_{j}$ ), and $\left(Z_{j}, \bar{Z}_{j}\right)$ is the associated complex frame given by

$$
Z_{j}=\frac{1}{2}\left(X_{j}-i Y_{j}\right), \quad \bar{Z}_{j}=\frac{1}{2}\left(X_{j}+i Y_{j}\right) .
$$

We also note that the dimension of an almost (pseudo-)Hermitian manifold is necessarily even (see e.g. [32]) and, in the case of a Kähler manifold, there is an intimate connection between its Kähler form and the volume form (which is just the Riemannian volume form determined by the metric) as follows

$$
d \mathcal{V}=\frac{1}{m !} \Omega^{m}
$$

where $d \mathcal{V}$ denotes the volume form of $M, \Omega^{m}$ is the wedge product of $\Omega$ with itself $m$ times, $m$ being the complex dimension of $M$ (see [4]). Hence the volume form is a real $(m, m)$-form on $M$.

On the other hand, if the volume of a Kähler manifold is written as

$$
d \mathcal{V}=d V \wedge d \bar{V}
$$

then $d V$ is the complex volume form of $M$. It is now clear that the complex volume form of a Kähler manifold can be written in a simple way with respect to any (pseudo-)orthonormal basis, using complex vierbeins $e_{i}+J e_{i}$. In fact, the complex volume form of a Kähler manifold $M$ is, up to a power factor of the imaginary unit $i$, the exterior product of these complex vierbeins.

\subsection{The Kähler cone of an Einstein-Sasaki manifold}

Let $M$ be a smooth manifold equipped with a triple $(\varphi, \xi, \eta)$, where $\varphi$ is a field of endomorphisms of the tangent spaces, $\xi$ is a vector field and $\eta$ is a 1 -form on $M$. If we have:

$$
\varphi^{2}=\tau(-I+\eta \otimes \xi), \quad \eta(\xi)=1,
$$

then we say that: 
(i) $(\varphi, \xi, \eta)$ is an almost contact structure on $M$, if $\tau=1$ (cf. [44]).

(ii) $(\varphi, \xi, \eta)$ is an almost paracontact structure on $M$, if $\tau=-1$ (cf. [45]).

A (pseudo-)Riemannian metric $g$ on $M$ is said to be compatible with the almost (para)contact structure $(\varphi, \xi, \eta)$ if and only if the relation

$$
g(\varphi X, \varphi Y)=\tau[g(X, Y)-\varepsilon \eta(X) \eta(Y)]
$$

holds for all pair of vector fields $X, Y$ on $M$, where $\varepsilon= \pm 1$, according as $\xi$ is space-like or time-like, respectively.

An almost (para)contact metric structure $(\varphi, \xi, \eta, g)$ is a (para-) Sasakian structure if and only if the Levi-Civita connection $\nabla$ of the metric $g$ satisfies

$$
\left(\nabla_{X} \varphi\right) Y=\tau[g(X, Y) \xi-\epsilon \eta(Y) X]
$$

for all vector fields $X, Y$ on $M$ (see [7]).

A (para-)Sasakian structure may also be reinterpreted and characterized in terms of the metric cone as follows. The (space-like) metric cone of a (pseudo-)Riemannian manifold $(M, g)$ is the (pseudo-)Riemannian manifold $C(M)=(0, \infty) \times M$ with the metric given by

$$
\bar{g}=d r^{2}+r^{2} g
$$

where $r$ is a coordinate on $(0, \infty)$. Then $M$ is a Sasaki manifold if and only if its metric cone $C(M)$ is Kähler [9] and we have a similar characterization for para-Sasakian manifolds [3]. In particular, the cone $C(M)$ is equipped with an integrable complex structure $J$ and a Kähler 2 -form $\Omega$, both of which are parallel with respect to the Levi-Civita connection $\bar{\nabla}$ of $\bar{g}$. Moreover, $M$ has odd dimension $2 n+1$, where $n+1$ is the complex dimension of the Kähler cone. We note that the Sasakian manifold $(M, g)$ is naturally isometrically embedded into the cone via the inclusion

$$
M=\{r=1\}=\{1\} \times M \subset C(M),
$$

and the Kähler structure of the cone $(C(M), \bar{g})$ induces an almost contact metric structure $(\phi, \xi, \eta, g)$ on $M$ satisfying (3).

A (para-)Einstein-Sasaki manifold is a Riemannian manifold $(M, g)$ that is both (para-)Sasaki and Einstein, i.e. a (para-)Sasakian manifold satisfying the Einstein condition

$$
\operatorname{Ric}_{g}=\lambda g
$$

for some real constant $\lambda$, where Ric $_{g}$ denotes the Ricci tensor of $g$. Einstein manifolds with $\lambda=0$ are called Ricci-flat manifolds. Similarly, an Einstein-Kähler manifold is a Riemannian manifold $(M, g)$ that is both Kähler and Einstein. The most important subclass of EinsteinKähler manifolds are the Calabi-Yau manifolds, which are Kähler and Ricci-flat.

It is also very important to note that the Gauss equation relating the curvature of submanifolds to the second fundamental form shows that a Sasaki manifold $M$ is Einstein if and only if the cone metric $C(M)$ is Kähler Ricci-flat. In particular the Kähler cone of an Einstein-Sasaki manifold has trivial canonical bundle and the restricted holonomy group of the cone is contained in $S U(m)$, where $m$ denotes the complex dimension of the Kähler cone [10, 49]. 


\section{Killing forms on Einstein-Sasaki spaces}

\subsection{Progression from Einstein-Kähler to Einstein-Sasaki to Calabi-Yau manifolds}

Suppose we have an Einstein-Sasaki metric $g_{E S}$ on a manifold $M_{2 n+1}$ of odd dimension $2 n+1$. An Einstein-Sasaki manifold can always be written as a fibration over an Einstein-Kähler manifold $M_{2 n}$ with the metric $g_{\mathrm{EK}}$ twisted by the overall $U(1)$ part of the connection [22]

$$
d s_{\mathrm{ES}}^{2}=\left(d \psi_{n}+2 A\right)^{2}+d s_{\mathrm{EK}}^{2}
$$

where $d A$ is given as the Kähler form of the Einstein-Kähler base. This can be easily seen when we write the metric of the cone manifold $M_{2 n+2}=C\left(M_{2 n+1}\right)$ as

$$
d s_{\text {cone }}^{2}=d r^{2}+r^{2} d s_{\mathrm{ES}}^{2}=d r^{2}+r^{2}\left(\left(d \psi_{n}+2 A\right)^{2}+d s_{\mathrm{EK}}^{2}\right) .
$$

The cone manifold is Calabi-Yau (i.e. Ricci flat and Kähler) and its Kähler form can be written as

$$
\Omega_{\text {cone }}=r d r \wedge\left(d \psi_{n}+2 A\right)+r^{2} \Omega_{\mathrm{EK}},
$$

and the Kähler condition $d \Omega_{\text {cone }}=0$ implies

$$
d A=\Omega_{\mathrm{EK}} .
$$

The Sasakian 1-form of the Einstein-Sasaki metric is

$$
\eta=2 A+d \psi_{n}
$$

which is a special unit-norm Killing 1-form obeying for all vector fields $X$ [46]

$$
\left.\nabla_{X} \eta=\frac{1}{2} X\right\lrcorner d \eta, \quad \nabla_{X}(d \eta)=-2 X^{*} \wedge \eta .
$$

\subsection{Kerr-NUT-(A)-dS space in a certain scaling limit}

In recent time new Einstein-Sasaki spaces have been constructed by taking certain BPS [18] or scaling limits [35, 38] of the Euclideanised Kerr-de Sitter metrics.

In even dimensions, performing the scaling limit on the Euclideanised Kerr-NUT-(A)dS spaces, the Einstein-Kähler metric $g_{\mathrm{EK}}$ and the Kähler potential $A$ are [35]:

$$
\begin{aligned}
& g_{\mathrm{EK}}=\frac{\Delta_{\mu} d x_{\mu}^{2}}{X_{\mu}}+\frac{X_{\mu}}{\Delta_{\mu}}\left(\sum_{j=0}^{n-1} \sigma_{\mu}^{(j)} d \psi_{j}\right)^{2}, \\
& X_{\mu}=-4 \prod_{i=1}^{n+1}\left(\alpha_{i}-x_{\mu}\right)-2 b_{\mu}, \quad A=\sum_{k=0}^{n-1} \sigma^{(k+1)} d \psi_{k},
\end{aligned}
$$

with

$$
\Delta_{\mu}=\prod_{\nu \neq \mu}\left(x_{\nu}-x_{\mu}\right), \quad \sigma_{\mu}^{(k)}=\sum_{\substack{\nu_{1}<\cdots<\nu_{k} \\ \nu_{i} \neq \mu}} x_{\nu_{1}} \cdots x_{\nu_{k}}, \quad \sigma^{(k)}=\sum_{\nu_{1}<\cdots<\nu_{k}} x_{\nu_{1}} \cdots x_{\nu_{k}} .
$$

Here, coordinates $x_{\mu}(\mu=1, \ldots, n)$ stands for the Wick rotated radial coordinate and longitudinal angles and the Killing coordinates $\psi_{k}(k=0, \ldots, n-1)$ denote time and azimuthal 
angles with Killing vectors $\xi^{(k)}=\partial_{\psi_{k}}$. Also $\alpha_{i}(i=1, \ldots, n+1)$ and $b_{\mu}$ are constants related to the cosmological constant, angular momenta, mass and NUT parameters [16].

We mention that in the case of odd-dimensional Kerr-NUT-(A)dS spaces the appropriate scaling limit leads to the same Einstein-Sasaki metric (4).

The hidden symmetries of the Sasaki manifold $M_{2 n+1}$ are described by the special Killing $(2 k+1)$-forms [46]:

$$
\Psi_{k}=\eta \wedge(d \eta)^{k}, \quad k=0,1, \ldots, n-1 .
$$

A sketch of this assertion is given in the appendix in a more general context.

Semmelmann obtained in [46] that special Killing forms on a Riemannian manifold $M$ are exactly those forms which translate into parallel forms on the metric cone $C(M)$. Therefore, the metric cone being either flat or irreducible, the problem of finding all special Killing forms is reduced to a holonomy problem (see [6]). In the case of holonomy $U(n+1)$, i.e. the cone $M_{2 n+2}=C\left(M_{2 n+1}\right)$ is Kähler, or equivalently $M_{2 n+1}$ is Sasaki, it follows that all special Killing forms are spanned by the forms $\Psi_{k}$ defined above. Besides these Killing forms, there are $n$ closed conformal Killing forms (also called $*$-Killing forms)

$$
\Phi_{k}=(d \eta)^{k}, \quad k=1, \ldots, n .
$$

Moreover, in the case of holonomy $S U(n+1)$, i.e. the cone $M_{2 n+2}=C\left(M_{2 n+1}\right)$ is Kähler and Ricci-flat, or equivalently $M_{2 n+1}$ is Einstein-Sasaki, it follows that we have two additional Killing forms of degree $n+1$ on the manifold $M_{2 n+1}$. These additional Killing forms are connected with the additional parallel forms of the Calabi-Yau cone manifold $M_{2 n+2}$ given by the complex volume form and its conjugate [46].

In order to write explicitly these additional Killing forms, we introduce the complex vierbeins on the Einstein-Kähler manifold $M_{2 n}$. First of all we shall write the metric $g_{\mathrm{EK}}$ in the form

$$
g_{\mathrm{EK}}=o^{\hat{\mu}} o^{\hat{\mu}}+\tilde{o}^{\hat{\mu}} \tilde{o}^{\hat{\mu}},
$$

and the Kähler 2-form

$$
\Omega=d A=o^{\hat{\mu}} \wedge \tilde{o}^{\hat{\mu}} .
$$

where

$$
o^{\hat{\mu}}=\sqrt{\frac{\Delta_{\mu}}{X_{\mu}\left(x_{\mu}\right)}} d x_{\mu}, \quad \tilde{o}^{\hat{\mu}}=\sqrt{\frac{X_{\mu}\left(x_{\mu}\right)}{\Delta_{\mu}}} \sum_{j=0}^{n-1} \sigma_{\mu}^{(j)} d \psi_{j} .
$$

We introduce the following complex vierbeins on Einstein-Kähler manifold $M_{2 n}$ :

$$
\zeta_{\mu}=o^{\hat{\mu}}+i \widetilde{o}^{\hat{\mu}}, \quad \mu=1, \ldots, n .
$$

On the Calabi-Yau cone manifold $M_{2 n+2}$ we take $\Lambda_{\mu}=r \zeta_{\mu}$ for $\mu=1, \ldots, n$ and

$$
\Lambda_{n+1}=\frac{d r}{r}+i \eta
$$

The standard complex volume form of the Calabi-Yau cone manifold [39] $M_{2 n+2}$ is

$$
d V=\Lambda_{1} \wedge \Lambda_{2} \wedge \cdots \wedge \Lambda_{n+1}
$$

As real forms we obtain the real respectively the imaginary part of the complex volume form. For example, writing

$$
\Lambda_{j}=\lambda_{2 j-1}+i \lambda_{2 j}, \quad j=1, \ldots, n+1,
$$


we obtain that the real part of the complex volume is given by

$$
\operatorname{Re} d V=\sum_{p=0}^{\left[\frac{n+1}{2}\right]} \sum_{\substack{1 \leq i_{1}<i_{2}<\cdots<i_{n+1} \leq 2 n+2 \\(C)}}(-1)^{p} \lambda_{i_{1}} \wedge \lambda_{i_{2}} \wedge \cdots \wedge \lambda_{i_{n+1}}
$$

where the condition $(C)$ in (6) means that in the second sum are taken only the indices $i_{1}, \ldots, i_{n+1}$ such that $i_{1}+\cdots+i_{n+1}=(n+1)^{2}+2 p$ and $\left(i_{k}, i_{k+1}\right) \neq(2 j-1,2 j)$, for all $k \in\{1, \ldots, n\}$ and $j \in\{1, \ldots, n+1\}$.

On the other hand, we obtain that the imaginary part of the complex volume is given by

$$
\operatorname{Im} d V=\sum_{p=0}^{\left[\frac{n}{2}\right]} \sum_{\substack{1 \leq i_{1}<i_{2}<\cdots<i_{n+1} \leq 2 n+2 \\\left(C^{\prime}\right)}}(-1)^{p} \lambda_{i_{1}} \wedge \lambda_{i_{2}} \wedge \cdots \wedge \lambda_{i_{n+1}}
$$

where the condition $\left(C^{\prime}\right)$ in $(7)$ means that in the second sum are considered only the indices $i_{1}, i_{2}, \ldots, i_{n+1}$ such that $i_{1}+\cdots+i_{n+1}=(n+1)^{2}+2 p+1$ and $\left(i_{k}, i_{k+1}\right) \neq(2 j-1,2 j)$, for all $k \in\{1, \ldots, n\}$ and $j \in\{1, \ldots, n+1\}$.

Finally, the Einstein-Sasaki manifold $M_{2 n+1}$ is identified with the submanifold $\{r=1\}$ of the Calabi-Yau cone manifold $M_{2 n+2}=C\left(M_{2 n+1}\right)$. In particular $\Lambda_{n+1}$ confines to its imaginary part and consequently, on the Einstein-Sasaki manifold $M_{2 n+1}$ we get

$$
\lambda_{2 n+1}=0, \quad \lambda_{2 n+2}=\eta
$$

and the additional $(n+1)$-Killing forms, denoted by $\Xi$ and $\Upsilon$ respectively, are accordingly acquired as follows:

$$
\Xi=\sum_{p=0}^{\left[\frac{n-1}{2}\right]} \sum_{\substack{1 \leq i_{1}<i_{2}<\cdots<i_{n} \leq 2 n \\\left(C_{1}\right)}}(-1)^{p+1} \lambda_{i_{1}} \wedge \lambda_{i_{2}} \wedge \cdots \wedge \lambda_{i_{n}} \wedge \eta,
$$

where the condition $\left(C_{1}\right)$ in (8) means that in the second sum are considered only the indices $i_{1}, i_{2}, \ldots, i_{n}$ such that $i_{1}+\cdots+i_{n}=n^{2}+2 p+1$ and $\left(i_{k}, i_{k+1}\right) \neq(2 j-1,2 j)$, for all $k \in$ $\{1, \ldots, n-1\}, j \in\{1, \ldots, n\}$, and

$$
\Upsilon=\sum_{p=0}^{\left[\frac{n}{2}\right]} \sum_{\substack{1 \leq i_{1}<i_{2}<\cdots<i_{n} \leq 2 n \\\left(C_{1}^{\prime}\right)}}(-1)^{p} \lambda_{i_{1}} \wedge \lambda_{i_{2}} \wedge \cdots \wedge \lambda_{i_{n}} \wedge \eta
$$

where the condition $\left(C_{1}^{\prime}\right)$ in $(9)$ means that in the second sum are taken only the indices $i_{1}, \ldots, i_{n}$ such that $i_{1}+\cdots+i_{n}=n^{2}+2 p$ and $\left(i_{k}, i_{k+1}\right) \neq(2 j-1,2 j)$, for all $k \in\{1, \ldots, n-1\}$ and $j \in\{1, \ldots, n\}$.

Moreover, in both relations (8) and (9), we have

$$
\lambda_{i_{k}}= \begin{cases}o^{\hat{j}}, & \text { if } i_{k}=2 j-1 \\ \tilde{o}^{\hat{j}}, & \text { if } i_{k}=2 j .\end{cases}
$$

\section{Conclusions}

In this paper we presented the complete set of Killing forms on Einstein-Sasaki spaces associated with Euclideanised Kerr-NUT-(A)dS spaces in a certain scaling limit. The multitude 
of Killing-Yano and Stäckel-Killing tensors makes possible a complete integrability of geodesic equations. In the case of geodesic and Klein-Gordon equations, the existence of separable coordinates is connected with Stäckel-Killing tensors. On the other hand from (conformal) Killing-Yano tensors one can construct first order differential operators which commute with Dirac operators [15]. In [13,41] it was shown that the solutions of Dirac equation in general higher dimensional Kerr-NUT-(A)dS spacetimes can be found by separating variables and the resulting ordinary differential equations can be completely decoupled. It is interesting to study separability and eigenvalues of Dirac operators on Einstein-Sasaki manifolds. Let us note also that in the higher dimensional Kerr-NUT-(A)dS spacetimes the stationary string equations are completely integrable [36]. An important open question is a separability problem for the gravitational perturbations in higher dimensional rotating black holes spacetimes, some preliminary results being achieved recently [42].

Another important direction of research is whether the Killing forms are also intrinsically linked to other higher spin perturbations. It is still an open question whether massless field equations, e.g. the Maxwell field, allow separation of variables in Kerr-NUT-(A)dS spaces.

These remarkable properties of higher dimensional black hole solutions offer new perspectives in investigation of hidden symmetries of other spacetimes structures. As a possible extension of these techniques we present in an appendix the case of spaces with mixed 3-structures which appear in many modern studies. Finally we mention some recent extensions of the Killing-Yano symmetry in the presence of skew-symmetric torsion. Preliminary results [24, 25] indicate that Killing forms in the presence of torsion preserve most of the properties of the standard Killing forms.

\section{A Killing forms on mixed 3-Sasakian manifolds}

The study of 3-Sasakian manifolds was initiated by Kuo [37] and presently there is an extensive literature on this topic (see for example [11] and references therein). It is well known that these manifolds are of great interest in physics, owing to their applications in supergravity and Mtheory $[1,2,23]$ and there exists a close relationship between quaternionic Kähler and 3-Sasakian structures [33]. On the other hand, the theory of paraquaternionic Kähler manifolds parallels the theory of quaternionic Kähler manifolds, but it uses the algebra of paraquaternionic numbers, in which two generators have square 1 and one generator has square -1 [21]. In what follows we recall some basic facts concerning this kind of structures, together with their closely linked counterpart in odd dimension (mixed 3-Sasakian structures).

An almost para-hypercomplex structure on a smooth manifold $M$ is a triple $H=\left(J_{1}, J_{2}, J_{3}\right)$ of $(1,1)$-type tensor fields on $M$ satisfying:

$$
J_{\alpha}^{2}=-\tau_{\alpha} \mathrm{Id}, \quad J_{\alpha} J_{\beta}=-J_{\beta} J_{\alpha}=\tau_{\gamma} J_{\gamma},
$$

for any $\alpha \in\{1,2,3\}$ and for any even permutation $(\alpha, \beta, \gamma)$ of $(1,2,3)$, where $\tau_{1}=\tau_{2}=-1=$ $-\tau_{3}$. In this case $(M, H)$ is said to be an almost para-hypercomplex manifold. A semi-Riemannian metric $g$ on $(M, H)$ is said to be compatible or adapted to the almost para-hypercomplex structure $H=\left(J_{\alpha}\right)_{\alpha=1,2,3}$ if it satisfies:

$$
g\left(J_{\alpha} X, J_{\alpha} Y\right)=\tau_{\alpha} g(X, Y)
$$

for all vector fields $X, Y$ on $M$ and $\alpha \in\{1,2,3\}$. Moreover, the pair $(g, H)$ is called an almost para-hyperhermitian structure on $M$ and the triple $(M, g, H)$ is said to be an almost para-hyperhermitian manifold. We note that any almost para-hyperhermitian manifold is of dimension $4 m, m \geq 1$, and any adapted metric is necessarily of neutral signature $(2 m, 2 m)$. If 
$\left\{J_{1}, J_{2}, J_{3}\right\}$ are parallel with respect to the Levi-Civita connection of $g$, then the manifold is called para-hyper-Kähler.

An almost paraquaternionic Hermitian manifold is a triple $(M, \sigma, g)$, where $M$ is a smooth manifold, $\sigma$ is an almost paraquaternionic structure on $M$, i.e. a rank 3-subbundle of $\operatorname{End}(T M)$ which is locally spanned by an almost para-hypercomplex structure $H=\left(J_{\alpha}\right)_{\alpha=1,2,3}$ and $g$ is a compatible metric with respect to $H$. If $(M, \sigma, g)$ is an almost paraquaternionic Hermitian manifold such that the bundle $\sigma$ is preserved by the Levi-Civita connection $\nabla$ of $g$, then $(M, \sigma, g)$ is said to be a paraquaternionic Kähler manifold [21]. We note that the prototype of paraquaternionic Kähler manifold is the paraquaternionic projective space $P^{n}(\widetilde{\mathbb{H}})$ as described by Blažić [8].

The counterpart in odd dimension of a paraquaternionic structure was introduced in [28] under the name of mixed 3-structure. This concept has been refined in [12], where the authors have introduced positive and negative metric mixed 3-structures. A mixed 3-structure on a smooth manifold $M$ is a triple of structures $\left(\varphi_{\alpha}, \xi_{\alpha}, \eta_{\alpha}\right), \alpha \in\{1,2,3\}$, which are almost paracontact structures for $\alpha=1,2$ and almost contact structure for $\alpha=3$, satisfying the following compatibility conditions

$$
\begin{aligned}
& \eta_{\alpha}\left(\xi_{\beta}\right)=0, \quad \varphi_{\alpha}\left(\xi_{\beta}\right)=\tau_{\beta} \xi_{\gamma}, \quad \varphi_{\beta}\left(\xi_{\alpha}\right)=-\tau_{\alpha} \xi_{\gamma}, \\
& \eta_{\alpha} \circ \varphi_{\beta}=-\eta_{\beta} \circ \varphi_{\alpha}=\tau_{\gamma} \eta_{\gamma}, \quad \varphi_{\alpha} \varphi_{\beta}-\tau_{\alpha} \eta_{\beta} \otimes \xi_{\alpha}=-\varphi_{\beta} \varphi_{\alpha}+\tau_{\beta} \eta_{\alpha} \otimes \xi_{\beta}=\tau_{\gamma} \varphi_{\gamma},
\end{aligned}
$$

where $(\alpha, \beta, \gamma)$ is an even permutation of $(1,2,3)$ and $\tau_{1}=\tau_{2}=-\tau_{3}=-1$.

Moreover, if a manifold $M$ with a mixed 3-structure $\left(\varphi_{\alpha}, \xi_{\alpha}, \eta_{\alpha}\right)_{\alpha=\overline{1,3}}$ admits a semi-Riemannian metric $g$ such that:

$$
g\left(\varphi_{\alpha} X, \varphi_{\alpha} Y\right)=\tau_{\alpha}\left[g(X, Y)-\varepsilon_{\alpha} \eta_{\alpha}(X) \eta_{\alpha}(Y)\right],
$$

for all $X, Y \in \Gamma(T M)$ and $\alpha=1,2,3$, where $\varepsilon_{\alpha}=g\left(\xi_{\alpha}, \xi_{\alpha}\right)= \pm 1$, then we say that $M$ has a metric mixed 3 -structure and $g$ is called a compatible metric.

In what follows a metric mixed 3 -structure will be denoted simply with $\left(\varphi_{\alpha}, \xi_{\alpha}, \eta_{\alpha}, g\right)$, leaving the condition $\alpha \in\{1,2,3\}$ understood. We note that if $\left(M, \varphi_{\alpha}, \xi_{\alpha}, \eta_{\alpha}, g\right)$ is a manifold with a metric mixed 3-structure then from (10) it follows

$$
g\left(\xi_{1}, \xi_{1}\right)=g\left(\xi_{2}, \xi_{2}\right)=-g\left(\xi_{3}, \xi_{3}\right) .
$$

Hence the vector fields $\xi_{1}$ and $\xi_{2}$ are both either space-like or time-like and these force the causal character of the third vector field $\xi_{3}$. We may therefore distinguish between positive and negative metric mixed 3-structures, according as $\xi_{1}$ and $\xi_{2}$ are both space-like, or both time-like vector fields. Because one can check that, at each point of $M$, there always exists a pseudoorthonormal frame field given by $\left\{\left(E_{i}, \varphi_{1} E_{i}, \varphi_{2} E_{i}, \varphi_{3} E_{i}\right)_{i=\overline{1, n}}, \xi_{1}, \xi_{2}, \xi_{3}\right\}$ we conclude that the dimension of the manifold is $4 n+3$ and the signature of $g$ is $(2 n+1,2 n+2)$, where we put first the minus signs, if the metric mixed 3 -structure is positive (i.e. $\varepsilon_{1}=\varepsilon_{2}=-\varepsilon_{3}=1$ ), or the signature of $g$ is $(2 n+2,2 n+1)$, if the metric mixed 3-structure is negative (i.e. $\varepsilon_{1}=\varepsilon_{2}=-\varepsilon_{3}=-1$ ).

A manifold $M$ endowed with a (positive/negative) metric mixed 3 -structure $\left(\varphi_{\alpha}, \xi_{\alpha}, \eta_{\alpha}, g\right)$ is said to be a (positive/negative) mixed 3-Sasakian structure if $\left(\varphi_{3}, \xi_{3}, \eta_{3}, g\right)$ is a Sasakian structure, while both structures $\left(\varphi_{1}, \xi_{1}, \eta_{1}, g\right)$ and $\left(\varphi_{2}, \xi_{2}, \eta_{2}, g\right)$ are para-Sasakian, i.e.

$$
\left(\nabla_{X} \varphi_{\alpha}\right) Y=\tau_{\alpha}\left[g(X, Y) \xi_{\alpha}-\epsilon_{\alpha} \eta_{\alpha}(Y) X\right]
$$

for all vector fields $X, Y$ on $M$ and $\alpha=1,2,3$.

It is important to note that, like their Riemannian counterparts, mixed 3-Sasakian structures are Einstein, but now the scalar curvature can be either positive or negative.

Theorem A.1 ([12,30]). Any $(4 n+3)$-dimensional manifold endowed with a mixed 3-Sasakian structure is an Einstein space with Einstein constant $\lambda=(4 n+2) \theta$, with $\theta=\mp 1$, according as the metric mixed 3-structure is positive or negative, respectively. 
We recall that the canonical example of manifold with negative mixed 3-Sasakian structure is the unit pseudo-sphere $S_{2 n+2}^{4 n+3} \subset \mathbb{R}_{2 n+2}^{4 n+4}$, while the pseudo-hyperbolic space $H_{2 n+1}^{4 n+3} \subset \mathbb{R}_{2 n+2}^{4 n+4}$ can be endowed with a canonical positive mixed 3-Sasakian structure. We also note that the existence of both positive and negative mixed 3-Sasakian structures in a principal $S O(2,1)$-bundle over a paraquaternionic Kähler manifold has been recently proved in [50].

Remark A.2. It is known [31] that on a mixed 3-Sasakian manifold $\left(M, \varphi_{\alpha}, \xi_{\alpha}, \eta_{\alpha}, g\right)$ of dimension $(4 n+3)$ there exists space-like, time-like and light-like Killing vector fields. Moreover, $\eta_{\alpha}$ are conformal Killing-Yano tensors of rank 1 on $M$, while $d \eta_{\alpha}$ are strictly conformal KillingYano tensors of rank 2 on $M$, for $\alpha=1,2,3$. On the other hand, the wedge products of $\eta_{\alpha}$ and $\left(d \eta_{\alpha}\right)^{k}$ provide Killing $(2 k+1)$-form, for $k=0,1, \ldots, 2 n+1$, since for any vector field $X$ on $M$ we have

$$
\begin{aligned}
\nabla_{X}\left(\eta_{\alpha} \wedge\left(d \eta_{\alpha}\right)^{k}\right) & =\nabla_{X} \eta_{\alpha} \wedge\left(d \eta_{\alpha}\right)^{k}+\eta_{\alpha} \wedge \nabla_{X}\left(d \eta_{\alpha}\right)^{k} \\
& \left.=\frac{1}{2}(X\lrcorner d \eta_{\alpha}\right) \wedge\left(d \eta_{\alpha}\right)^{k}+k \eta_{\alpha} \wedge \nabla_{X} d \eta_{\alpha} \wedge\left(d \eta_{\alpha}\right)^{k-1} \\
& \left.=\frac{1}{2(k+1)} X\right\lrcorner\left(d \eta_{\alpha}\right)^{k+1}-\frac{k}{4 n+2} \eta_{\alpha} \wedge\left(X^{*} \wedge d^{*}\left(d \eta_{\alpha}\right)\right) \wedge\left(d \eta_{\alpha}\right)^{k-1} \\
& \left.\left.=\frac{1}{2(k+1)} X\right\lrcorner\left(d \eta_{\alpha}\right)^{k+1}=\frac{1}{2(k+1)} X\right\lrcorner d\left(\eta_{\alpha} \wedge\left(d \eta_{\alpha}\right)^{k}\right) .
\end{aligned}
$$

It follows from a simple computation that the wedge product of $\eta_{\alpha}$ and $\left(d \eta_{\alpha}\right)^{k}$ provides a special Killing form, since it satisfies the additional equation

$$
\nabla_{X}\left(d\left(\eta_{\alpha} \wedge\left(d \eta_{\alpha}\right)^{k}\right)\right)=-2(k+1) X^{*} \wedge \eta_{\alpha} \wedge\left(d \eta_{\alpha}\right)^{k}
$$

for any vector field $X$ on $M$. Therefore, as in 3-Sasakian case [46], we obtain that any linear combination of the forms $\Psi_{k_{1}, k_{2}, k_{3}}$ defined by

$$
\begin{aligned}
\Psi_{k_{1}, k_{2}, k_{3}}= & \frac{k_{1}}{k_{1}+k_{2}+k_{3}}\left[\eta_{1} \wedge\left(d \eta_{1}\right)^{k_{1}-1}\right] \wedge\left(d \eta_{2}\right)^{k_{2}} \wedge\left(d \eta_{3}\right)^{k_{3}} \\
& +\frac{k_{2}}{k_{1}+k_{2}+k_{3}}\left(d \eta_{1}\right)^{k_{1}} \wedge\left[\eta_{2} \wedge\left(d \eta_{2}\right)^{k_{2}-1}\right] \wedge\left(d \eta_{3}\right)^{k_{3}} \\
& +\frac{k_{3}}{k_{1}+k_{2}+k_{3}}\left(d \eta_{1}\right)^{k_{1}} \wedge\left(d \eta_{2}\right)^{k_{2}} \wedge\left[\eta_{3} \wedge\left(d \eta_{3}\right)^{k_{3}-1}\right],
\end{aligned}
$$

for arbitrary positive integers $k_{1}, k_{2}, k_{3}$, is a special Killing form on $M$. The special Killing forms (5) could be recovered as a particular case of (12) for two vanishing integers $k_{i}$.

Remark A.3. For the rest of this section we consider that $(\bar{M}, \sigma, \bar{g})$ is an almost paraquaternionic Hermitian manifold of dimension $(4 n+4)$ and $(M, g)$ is an orientable non-degenerate hypersurface of $M$ with $g=\bar{g}_{\mid M}$, such that the normal bundle $T M^{\perp}$ is generated by a unit space-like or time-like vector field $\xi$ normal to $M$. Then for any vector field $X$ on $M$ and any local basis $H=\left(J_{\alpha}\right)_{\alpha=1,2,3}$ of $\sigma$, we have the decomposition

$$
J_{\alpha} X=\varphi_{\alpha} X+F_{\alpha} X
$$

for $\alpha \in\{1,2,3\}$, where $\varphi_{\alpha} X$ and $F_{\alpha} X$ are the tangent part and the normal part of $J_{\alpha} X$, respectively. We can remark that, in fact, $F_{\alpha} X \in \Gamma\left(T M^{\perp}\right)$ for any vector field $X$ on $M$, and therefore we deduce the decomposition

$$
J_{\alpha} X=\varphi_{\alpha} X+\eta_{\alpha}(X) \xi
$$


where

$$
\eta_{\alpha}(X)=-\varepsilon \bar{g}\left(X, J_{\alpha} \xi\right), \quad \varepsilon=g(\xi, \xi) .
$$

If we define now the vector field $\xi_{\alpha}$ by

$$
\xi_{\alpha}=-\tau_{\alpha} J_{\alpha} \xi
$$

for $\alpha \in\{1,2,3\}$, then we obtain by direct computations that the paraquaternionic structure $\sigma$ on $\bar{M}$ induces a positive/negative metric mixed 3 -structure $\left(\varphi_{\alpha}, \xi_{\alpha}, \eta_{\alpha}, g\right)$ on $M$ as follows (see [29] for the proof in the case of negative metric mixed 3-structure).

Theorem A.4. Let $(M, g)$ be an orientable non-degenerate hypersurface of an almost paraquaternionic Hermitian manifold $(\bar{M}, \sigma, \bar{g})$ with the normal bundle $T M^{\perp}$ spanned by a unit space-like or time-like normal vector field $\xi$. Then $\left(\varphi_{\alpha}, \xi_{\alpha}, \eta_{\alpha}, g\right)$ defined above is a positive/negative metric mixed 3-structure on $M$, according as the generator $\xi$ is a time-like or a space-like vector field.

We recall now that if $\bar{\nabla}$ is the Levi-Civita connection on $\bar{M}$ and denote by $\nabla$ the Levi-Civita connection induced on $M$, then the Gauss and Weingarten formulas are given by

$$
\bar{\nabla}_{X} Y=\nabla_{X} Y+h(X, Y) \xi \quad \text { and } \quad \bar{\nabla}_{X} \xi=-A X,
$$

for all vector fields $X, Y$ tangent to $M$, where $h$ is the second fundamental form of $M$ and $A$ is the fundamental tensor of Weingarten with respect to the unit space-like or time-like normal vector field $\xi$.

From (13) we deduce

$$
\varepsilon h(X, Y)=g(A X, Y)
$$

for all $X, Y \in \Gamma(T M)$, where $\varepsilon=g(\xi, \xi)$.

Theorem A.5. Let $M$ be an orientable non-degenerate hypersurface of a para-hyper-Kähler manifold $\left(\bar{M}, H=\left(J_{1}, J_{2}, J_{3}\right), \bar{g}\right)$ and let $\left(\varphi_{\alpha}, \xi_{\alpha}, \eta_{\alpha}, g\right)$ be the canonical metric mixed 3 -structure on $M$. Then:

(i) $\eta_{1}, \eta_{2}, \eta_{3}$ are Killing if and only if

$$
h\left(X, \varphi_{\alpha} Y\right)=-h\left(\varphi_{\alpha} X, Y\right), \quad \alpha=1,2,3 ;
$$

(ii) $\varphi_{1}, \varphi_{2}, \varphi_{3}$ are covariant constant, provided that $M$ is a totally geodesic hypersurface of $\bar{M}$;

(iii) $\varphi_{\alpha}$ is Killing if and only if $h$ is proportional to $\eta_{\alpha} \otimes \eta_{\alpha}, \alpha=1,2,3$, provided that $J_{1}, J_{2}, J_{3}$ are Killing.

Proof. Since each $J_{\alpha}$ is parallel with respect to the Levi-Civita connection of $\bar{g}$, then using (13) and (14) we obtain

$$
\begin{aligned}
0 & =\left(\bar{\nabla}_{X} J_{\alpha}\right) Y=\bar{\nabla}_{X} J_{\alpha} Y-J_{\alpha} \bar{\nabla}_{X} Y=\bar{\nabla}_{X}\left(\varphi_{\alpha} Y+\eta_{\alpha}(Y) \xi\right)-J_{\alpha}\left(\nabla_{X} Y+h(X, Y) \xi\right) \\
& =\left(\nabla_{X} \varphi_{\alpha}\right) Y+\tau_{\alpha} h(X, Y) \xi_{\alpha}-\eta_{\alpha}(Y) A X+\left[h\left(X, \varphi_{\alpha} Y\right)+\left(\nabla_{X} \eta_{\alpha}\right) Y\right] \xi
\end{aligned}
$$

for all vector fields $X, Y$ on $M$. Taking now the tangential and the normal component of both sides of the above equation we deduce

$$
\left(\nabla_{X} \varphi_{\alpha}\right) Y=-\tau_{\alpha} h(X, Y) \xi_{\alpha}+\eta_{\alpha}(Y) A X,
$$

and

$$
\left(\nabla_{X} \eta_{\alpha}\right) Y=-h\left(X, \varphi_{\alpha} Y\right) .
$$

The proof of the assertions $(i),(i i)$ and (iii) follows now easily using (15) and (16) with some standard algebraic manipulations. 
Next we suppose that $M$ is a non-degenerate totally umbilical hypersurface of a para-hyperKähler manifold $\left(\bar{M}, H=\left(J_{1}, J_{2}, J_{3}\right), \bar{g}\right)$, i.e. for all vector fields $X, Y$ on $M$ we have

$$
h(X, Y)=\lambda g(X, Y),
$$

for some function $\lambda$. Now we are able to prove that the canonical metric mixed 3-structure on $M$ can be a positive or a negative mixed 3-Sasakian structure in some conditions (compare with [48] for the corresponding result in quaternionic setting).

Theorem A.6. Let $M$ be an orientable non-degenerate hypersurface of a para-hyper-Kähler manifold $\left(\bar{M}, H=\left(J_{1}, J_{2}, J_{3}\right), \bar{g}\right)$ with the normal bundle $T M^{\perp}$ spanned by a unit space-like or time-like vector field $\xi$. If $M$ is a totally umbilical hypersurface of $\bar{M}$, then:

(i) the canonical metric mixed 3-structure $\left(\varphi_{\alpha}, \xi_{\alpha}, \eta_{\alpha}, g\right)$ on $M$ is a positive mixed 3-Sasakian structure if and only if $\lambda=-1$ and $\xi$ is time-like;

(ii) the canonical metric mixed 3-structure $\left(\varphi_{\alpha}, \xi_{\alpha}, \eta_{\alpha}, g\right)$ on $M$ is a negative mixed 3-Sasakian structure if and only if $\lambda=-1$ and $\xi$ is space-like.

Proof. Using (17) in (15) we obtain

$$
\left(\nabla_{X} \varphi_{\alpha}\right) Y=-\tau_{\alpha} \lambda g(X, Y) \xi_{\alpha}+\eta_{\alpha}(Y) A X,
$$

for all vector fields $X, Y$ on $M$.

On the other hand, from (14) and (17) we derive

$$
A X=\varepsilon \lambda X,
$$

for any vector field $X$ on $M$.

From (18) and (19) we deduce

$$
\left(\nabla_{X} \varphi_{\alpha}\right) Y=-\lambda \tau_{\alpha}\left[g(X, Y) \xi_{\alpha}-\frac{\varepsilon}{\tau_{\alpha}} \eta_{\alpha}(Y) X\right],
$$

for all vector fields $X, Y$ on $M$.

(i) Taking now into account that in the case of a positive metric mixed 3-structure we have $\varepsilon_{\alpha} \tau_{\alpha}=-1$, for $\alpha=1,2,3$, we obtain that the equation (20) can be rewritten as

$$
\left(\nabla_{X} \varphi_{\alpha}\right) Y=-\lambda \tau_{\alpha}\left[g(X, Y) \xi_{\alpha}+\varepsilon \varepsilon_{\alpha} \eta_{\alpha}(Y) X\right] .
$$

Comparing (11) with (21) we deduce that the canonical metric mixed 3 -structure $\left(\varphi_{\alpha}, \xi_{\alpha}, \eta_{\alpha}, g\right)$ on $M$ is a positive mixed 3-Sasakian structure if and only if $\lambda=-1$ and $\varepsilon=-1$, and the assertion is now clear.

(ii) Since in the case of a negative metric mixed 3-structure we have $\varepsilon_{\alpha} \tau_{\alpha}=1$, for $\alpha=1,2,3$, we can rewrite (20) in the following form:

$$
\left(\nabla_{X} \varphi_{\alpha}\right) Y=-\lambda \tau_{\alpha}\left[g(X, Y) \xi_{\alpha}-\varepsilon \varepsilon_{\alpha} \eta_{\alpha}(Y) X\right] .
$$

Comparing now (11) and (22) we deduce that the canonical metric mixed 3-structure $\left(\varphi_{\alpha}, \xi_{\alpha}\right.$, $\left.\eta_{\alpha}, g\right)$ on $M$ is a negative mixed 3-Sasakian structure if and only if $\lambda=-1$ and $\varepsilon=1$, and the conclusion follows.

\section{Acknowledgments}

The authors would like to thank to the referees for comments and valuable suggestions. MV was supported by CNCS-UEFISCDI, project number PN-II-ID-PCE-2011-3-0137. The work of GEV was supported by CNCS-UEFISCDI, project number PN-II-ID-PCE-2011-3-0118. 


\section{References}

[1] Acharya B.S., Figueroa-O'Farrill J.M., Hull C.M., Spence B., Branes at conical singularities and holography, Adv. Theor. Math. Phys. 2 (1998), 1249-1286, hep-th/9808014.

[2] Agricola I., Friedrich T., Killing spinors in supergravity with 4-fluxes, Classical Quantum Gravity 20 (2003), 4707-4717, math.DG/0307360.

[3] Alekseevsky D.V., Cortés V., Galaev A.S., Leistner T., Cones over pseudo-Riemannian manifolds and their holonomy, J. Reine Angew. Math. 635 (2009), 23-69, arXiv:0707.3063.

[4] Ballmann W., Lectures on Kähler manifolds, ESI Lectures in Mathematics and Physics, European Mathematical Society (EMS), Zürich, 2006.

[5] Bär C., Real Killing spinors and holonomy, Comm. Math. Phys. 154 (1993), 509-521.

[6] Besse A.L., Einstein manifolds, Ergebnisse der Mathematik und ihrer Grenzgebiete (3), Vol. 10, SpringerVerlag, Berlin, 1987.

[7] Blair D.E., Contact manifolds in Riemannian geometry, Lecture Notes in Mathematics, Vol. 509, SpringerVerlag, Berlin, 1976.

[8] Blažić N., Paraquaternionic projective space and pseudo-Riemannian geometry, Publ. Inst. Math. (Beograd) (N.S.) 60 (1996), 101-107.

[9] Boyer C., Galicki K., 3-Sasakian manifolds, in Surveys in Differential Geometry: Essays on Einstein Manifolds, Surv. Differ. Geom., Vol. VI, Int. Press, Boston, MA, 1999, 123-184, hep-th/9810250.

[10] Boyer C.P., Galicki K., Sasakian geometry, holonomy, and supersymmetry, in Handbook of pseudoRiemannian geometry and supersymmetry, IRMA Lect. Math. Theor. Phys., Vol. 16, Eur. Math. Soc., Zürich, 2010, 39-83, math.DG/0703231.

[11] Boyer C.P., Galicki K., Mann B.M., The geometry and topology of 3-Sasakian manifolds, J. Reine Angew. Math. 455 (1994), 183-220.

[12] Caldarella A.V., Pastore A.M., Mixed 3-Sasakian structures and curvature, Ann. Polon. Math. 96 (2009), 107-125, arXiv:0803.1953.

[13] Cariglia M., Krtouš P., Kubizñák D., Dirac equation in Kerr-NUT-(A)dS spacetimes: intrinsic characterization of separability in all dimensions, Phys. Rev. D 84 (2011), 024008, 11 pages, arXiv:1104.4123.

[14] Carter B., Killing tensor quantum numbers and conserved currents in curved space, Phys. Rev. D 16 (1977), 3395-3414.

[15] Carter B., McLenaghan R.G., Generalized total angular momentum operator for the Dirac equation in curved space-time, Phys. Rev. D 19 (1979), 1093-1097.

[16] Chen W., Lü H., Pope C.N., General Kerr-NUT-AdS metrics in all dimensions, Classical Quantum Gravity 23 (2006), 5323-5340, hep-th/0604125.

[17] Cortés V., Mayer C., Mohaupt T., Saueressig F., Special geometry of Euclidean supersymmetry. II. Hypermultiplets and the c-map, J. High Energy Phys. 2005 (2005), no. 6, 025, 37 pages, hep-th/0503094.

[18] Cvetič M., Lü H., Page D.N., Pope C.N., New Einstein-Sasaki and Einstein spaces from Kerr-de Sitter, J. High Energy Phys. 2009 (2009), no. 7, 082, 25 pages, hep-th/0505223.

[19] Dunajski M., West S., Anti-self-dual conformal structures in neutral signature, in Recent developments in pseudo-Riemannian geometry, ESI Lect. Math. Phys., Eur. Math. Soc., Zürich, 2008, 113-148, math.DG/0610280.

[20] Emparan R., Reall H.S., Black holes in higher dimensions, Living Rev. Relativ. 11 (2008), 6, 87 pages, arXiv:0801.3471.

[21] García-Río E., Matsushita Y., Vázquez-Lorenzo R., Paraquaternionic Kähler manifolds, Rocky Mountain J. Math. 31 (2001), 237-260.

[22] Gibbons G.W., Hartnoll S.A., Pope C.N., Bohm and Einstein-Sasaki metrics, black holes, and cosmological event horizons, Phys. Rev. D 67 (2003), 084024, 24 pages, hep-th/0208031.

[23] Gibbons G.W., Rychenkova P., Cones, tri-Sasakian structures and superconformal invariance, Phys. Lett. B 443 (1998), 138-142, hep-th/9809158.

[24] Houri T., Kubizňák D., Warnick C.M., Yasui Y., Generalized hidden symmetries and the Kerr-Sen black hole, J. High Energy Phys. 2010 (2010), no. 7, 055, 33 pages, arXiv:1004.1032.

[25] Houri T., Kubizńák D., Warnick C., Yasui Y., Symmetries of the Dirac operator with skew-symmetric torsion, Classical Quantum Gravity 27 (2010), 185019, 16 pages, arXiv:1002.3616. 
[26] Hull C.M., Actions for $(2,1)$ sigma models and strings, Nuclear Phys. B 509 (1998), 252-272, hep-th/9702067.

[27] Hull C.M., Duality and the signature of space-time, J. High Energy Phys. 1998 (1998), no. 11, 017, 36 pages, hep-th/9807127.

[28] Ianuş S., Mazzocco R., Vîlcu G.E., Real lightlike hypersurfaces of paraquaternionic Kähler manifolds, Mediterr. J. Math. 3 (2006), 581-592.

[29] Ianuş S., Vîlcu G.E., Paraquaternionic manifolds and mixed 3-structures, in Differential Geometry, World Sci. Publ., Hackensack, NJ, 2009, 276-285.

[30] Ianuş S., Vîlcu G.E., Some constructions of almost para-hyperhermitian structures on manifolds and tangent bundles, Int. J. Geom. Methods Mod. Phys. 5 (2008), 893-903, arXiv:0707.3360.

[31] Ianuş S., Visinescu M., Vîlcu G.E., Conformal Killing-Yano tensors on manifolds with mixed 3-structures, SIGMA 5 (2009), 022, 12 pages, arXiv:0902.3968.

[32] Kobayashi S., Nomizu K., Foundations of differential geometry, Vol. I, Interscience Publishers, New YorkLondon, 1963.

[33] Konishi M., On manifolds with Sasakian 3-structure over quaternion Kaehler manifolds, Kōdai Math. Sem. Rep. 26 (1974/75), 194-200.

[34] Krtouš P., Kubizňák D., Page D.N., Vasudevan M., Constants of geodesic motion in higher-dimensional black-hole spacetimes, Phys. Rev. D $\mathbf{7 6}$ (2007), 084034, 8 pages, arXiv:0707.0001.

[35] Kubizňák D., On the supersymmetric limit of Kerr-NUT-AdS metrics, Phys. Lett. B 675 (2009), 110-115, arXiv:0902.1999.

[36] Kubizňák D., Frolov V.P., Stationary strings and branes in the higher-dimensional Kerr-NUT-(A)dS spacetimes, J. High Energy Phys. 2008 (2008), no. 2, 007, 14 pages, arXiv:0711.2300.

[37] Kuo Y., On almost contact 3-structure, Tôhoku Math. J. 22 (1970), 325-332.

[38] Martelli D., Sparks J., Toric Sasaki-Einstein metrics on $S^{2} \times S^{3}$, Phys. Lett. B 621 (2005), 208-212, hep-th/0505027.

[39] Ohnita Y., Stability and rigidity of special Lagrangian cones over certain minimal Legendrian orbits, Osaka J. Math. 44 (2007), 305-334.

[40] Ooguri H., Vafa C., Geometry of $N=2$ strings, Nuclear Phys. B 361 (1991), 469-518.

[41] Oota T., Yasui Y., Separability of Dirac equation in higher dimensional Kerr-NUT-de Sitter spacetime, Phys. Lett. B 659 (2008), 688-693, arXiv:0711.0078.

[42] Oota T., Yasui Y., Separability of gravitational perturbation in generalized Kerr-NUT-de Sitter space-time, Internat. J. Modern Phys. A 25 (2010), 3055-3094, arXiv:0812.1623.

[43] Page D.N., Kubizñák D., Vasudevan M., Krtouš P., Complete integrability of geodesic motion in general higher-dimensional rotating black-hole spacetimes, Phys. Rev. Lett. 98 (2007), 061102, 4 pages, hep-th/0611083.

[44] Sasaki S., On differentiable manifolds with certain structures which are closely related to almost contact structure. I, Tôhoku Math. J. 12 (1960), 459-476.

[45] Satō I., On a structure similar to the almost contact structure, Tensor (N.S.) 30 (1976), 219-224.

[46] Semmelmann U., Conformal Killing forms on Riemannian manifolds, Math. Z. 245 (2003), 503-527, math.DG/0206117.

[47] Sergyeyev A., Krtouš P., Complete set of commuting symmetry operators for the Klein-Gordon equation in generalized higher-dimensional Kerr-NUT-(A)dS spacetimes, Phys. Rev. D 77 (2008), 044033, 6 pages, arXiv:0711.4623.

[48] Sharfuddin A., Shahid M.H., Hypersurfaces of almost quaternion manifolds, Soochow J. Math. 20 (1994), 297-308.

[49] Sparks J., Sasaki-Einstein manifolds, in Geometry of Special Holonomy and Related Topics, Surv. Differ. Geom., Vol. 16, Int. Press, Somerville, MA, 2011, 265-324, arXiv:1004.2461.

[50] Vîlcu G.E., Voicu R.C., Curvature properties of pseudo-sphere bundles over paraquaternionic manifolds, Int. J. Geom. Methods Mod. Phys. 9 (2012), 1250024, 23 pages, arXiv:1107.5260.

[51] Yano K., Some remarks on tensor fields and curvature, Ann. of Math. (2) 55 (1952), 328-347. 